\title{
An Efficient Uniform-Cost Normalized Edit Distance Algorithm
}

\author{
Abdullah N. Arslan and Ömer Eḡecioḡlu \\ Department of Computer Science \\ University of California, Santa Barbara \\ \{arslan, omer\}@cs.ucsb.edu
}

\begin{abstract}
A common model for computing the similarity of two strings $X$ and $Y$ of lengths $m$, and $n$ respectively with $m \geq n$, is to transform $X$ into $Y$ through a sequence of three types of edit operations: insertion, deletion, and substitution. The model assumes a given cost function which assigns a non-negative real weight to each edit operation. The amortized weight for a given edit sequence is the ratio of its weight to its length, and the minimum of this ratio over all edit sequences is the normalized edit distance. Existing algorithms for normalized edit distance computation with proven complexity bounds require $O\left(m n^{2}\right)$ time in the worst-case. We give an $O(m n \log n)$-time algorithm for the problem when the cost function is uniform, i.e, the weight of each edit operation is constant within the same type, except substitutions can have different weights depending on whether they are matching or non-matching.
\end{abstract}

Keywords: Edit distance, normalized edit distance, algorithm, dynamic programming, fractional programming, $r a-$ tio minimization.

\section{Introduction}

Measuring similarity of strings is a well-known problem in computer science which has applications in many fields such as computational biology, text processing, optical character recognition, image and signal processing, error correction, information retrieval, pattern recognition, and pattern matching in large databases.

Consider two strings $X$ and $Y$ over a finite alphabet whose lengths are $m$ and $n$ respectively with $m \geq n$. We consider sequences of weighted edit operations (insertions, deletions, and substitutions of characters), by means of which $X$ is transformed into $Y$. If we call each such sequence an edit sequence, then the ordinary (conventional) edit distance problem (ED) seeks for an edit sequence with minimum total weight over all sequences. The edit distance between $X$ and $Y$ is defined as the weight of such a sequence. Although the edit distance is a useful measure for similarity of two strings, for some applications the lengths of the strings compared need to be taken into account. Two binary strings of length 1000 differing in 1 bit have the same edit distance as two binary strings of length 2 differing in 1 bit, although one would most likely state that only the 1000-bit strings are "almost equal". It is tempting to normalize the edit distance by an appropriate method: Marzal and Vidal considered a variant called normalized edit distance (NED) between $X$ and $Y$ [7]. If we define the amortized weight of an edit sequence as the ratio of the total weight of the sequence to the length of the sequence, NED is the minimum amortized weight over all edit sequences. Marzal and Vidal found that NED yields better results in empirical experiments. However, it seems that the computation of NED requires significantly more work than that of ordinary edit distance algorithms, which are mostly dynamic programming based. It is also interesting to note that NED cannot be obtained by "post-normalization", i.e., first computing the ordinary edit distance and then dividing it by the length of the corresponding edit sequence [7].

Ordinary edit distance can be computed in $O(m n)$ time $[13,17,5]$, or $O(m n / \log n)$ time if the weights are rational [8]. In order for NED computations to be advantageous, the computational complexity of an algorithm for the latter should not significantly exceed these. There are several algorithms to compute NED, both sequential $[7,11,16]$ and parallel [4]. Observing that an edit sequence length lies in the range $m$ and $m+n$ inclusive, an $O\left(m n^{2}\right)$-time dynamic programming algorithm can be developed for this problem [7]. Furthermore, it has been noted that NED can be formulated as a special case of constrained edit distance (CED) problems [11]. By adapting the techniques used for CED, NED can be computed in $O(s m n)$ time where $s$ is the number of substitutions in an optimal edit sequence [11]. But since $s$ can be as large as $n$, the worst case time complexity remains $O\left(m n^{2}\right)$.

Another approach for NED computation uses fractional programming [16]; an iterative method in which an ordi- 
nary edit distance problem with varying weights is solved at each iteration. Experimental results on both randomly generated synthetic data, and real applications performed by Vidal, Marzal, and Aibar [16] suggest that the number of iterations necessary for the NED computation with this method is bounded by a small constant. This implies an achievement of experimental $O(m n)$ time complexity for NED computation, but as argued by Vidal, Marzal, and Aibar, a mathematical proof of a theoretical bound for this algorithm seems difficult. However, it may be possible to analyze at least the average complexity under a reasonable probabilistic model.

In this paper, we direct our attention to NED computations in the case when the cost function is uniform; i.e. weight of an edit operation does not depend on the symbols involved in the operation, but only on its type. Uniform and even more restricted cases of cost functions have been studied in the literature in the context of ordinary edit distances $[12,15,10]$. We assume four types of operations: insertion, deletion, matching and non-matching substitutions.

The previously suggested algorithms for NED do not specialize to a faster than $O\left(m n^{2}\right)$-time algorithm when the cost function is uniform. We propose an algorithm UniformNED which iteratively solves ordinary edit distance problems to compute the NED. We use the standard algorithm of Wagner and Fisher [17] to solve the ordinary edit distance problems that arise, and make use of a technique developed by Megiddo [9] for the optimization of objective functions which are ratios of linear functions. The worst-case resulting time complexity of our algorithm is $O(m n \log n)$, compared to the best provable complexity of $O\left(m n^{2}\right)$ of previously known NED algorithms.

The outline of this paper is as follows. Section 2 consists of preliminaries; definitions, notation used, and the problem statement. In section 3 we describe optimization of the ratio of two linear functions and Megiddo's method. Section 4 describes our algorithm and gives a proof of its $O(m n \log n)$ worst-case time complexity, and section 5 describes its implementation. This is followed by remarks in section 6 and conclusions in section 7.

\section{Definitions}

Let $X=x_{1} x_{2} \cdots x_{m}$ and $Y=y_{1} y_{2} \cdots y_{n}$ be two strings over an alphabet $\Sigma$ for $m \geq 0$, and $n \geq 0$, not both null. We assume that the edit operations applicable on the symbols of $X$ to transform it into $Y$ are of three types: inserting a character into $X$, deleting a character from $X$, or substituting a character from $\Sigma$ for a character of $X$. The substitution operation can further be broken down into matching and non-matching substitutions. More formally for $1 \leq i \leq m$, the allowable edit operations are

(1) Insertion: any symbol $s \in \Sigma$ can be inserted before or after $x_{i}$,

(2) Deletion: the symbol $x_{i}$ can be deleted.

(3) Substitution: the symbol $x_{i}$ can be replaced by a symbol $s \in \Sigma$. A substitution operation is

(3-a) a matching substitution if $s=x_{i}$,

(3-b) a non-matching substitution if $s \neq x_{i}$.

The edit graph $G_{X, Y}$ of $X$ and $Y$ is a directed acyclic graph having $(m+1)(n+1)$ lattice points $(i, j)$ for $0 \leq i \leq$ $m$, and $0 \leq j \leq n$ as vertices. The top-left extreme point of this rectangular grid is labeled $(0,0)$ and the bottom-right extreme point is labeled $(m, n)$, as shown in Figure 1. The arcs of $G_{X, Y}$ are divided into three types corresponding to edit operations:

(1) Horizontal arcs: $\{((i, j-1),(i, j)) \mid 0 \leq i \leq m$, $0<j \leq n\}$ (deletions).

(2) Vertical arcs: $\{((i-1, j),(i, j)) \mid 0<i \leq m, 0 \leq$ $j \leq n\}$ (insertions).

(3) Diagonal arcs: $\{((i-1, j-1),(i, j)) \mid 0<i \leq m$, $0<j \leq n\}$ (substitutions).

If $x_{i}=y_{j}$, then the diagonal arc $((i-1, j-1),(i, j))$ is a matching diagonal arc, otherwise a non-matching diagonal $a r c$. Figure 1 illustrates an example edit graph for $X=a b a$ and $Y=b a b$.

An edit path in $G_{X, Y}$ is a directed path from $(0,0)$ to $(m, n)$. Steps of an edit path correspond to a sequence of edit operations which transforms $X$ into $Y$ as follows: A horizontal arc $((i-1, j),(i, j))$ corresponds to the deletion of $x_{i}$, a vertical arc $((i-1, j-1),(i-1, j))$ corresponds to the insertion of $y_{j}$ immediately before $x_{i}$, and a diagonal arc $((i-1, j-1),(i, j))$ corresponds to substitution of symbol $y_{j}$ for $x_{i}$. In Figure 1, horizontal, vertical, and diagonal arcs are labeled by the initial letters of the corresponding edit operations $D, I$, and $S$, respectively. Matching diagonal arcs are indicated by dashed lines, whereas non-matching diagonal arcs are straight lines.

Ordinarily a cost function for uniform weights is a triple of non-negative real numbers specifying the costs of insertion, deletion, and non-matching substitution. Generally the cost of a matching substitution is assumed to be zero. In our case we use a refinement which is defined by a 4tuple of non-negative real numbers $\gamma=\left(\gamma_{I}, \gamma_{D}, \gamma_{M}, \gamma_{N}\right)$. These specify the cost of an insertion $\left(\gamma_{I}\right)$, deletion $\left(\gamma_{D}\right)$, matching substitution $\left(\gamma_{M}\right)$, and non-matching substitution $\left(\gamma_{N}\right)$. This turns $G_{X, Y}$ into a weighted graph in which 


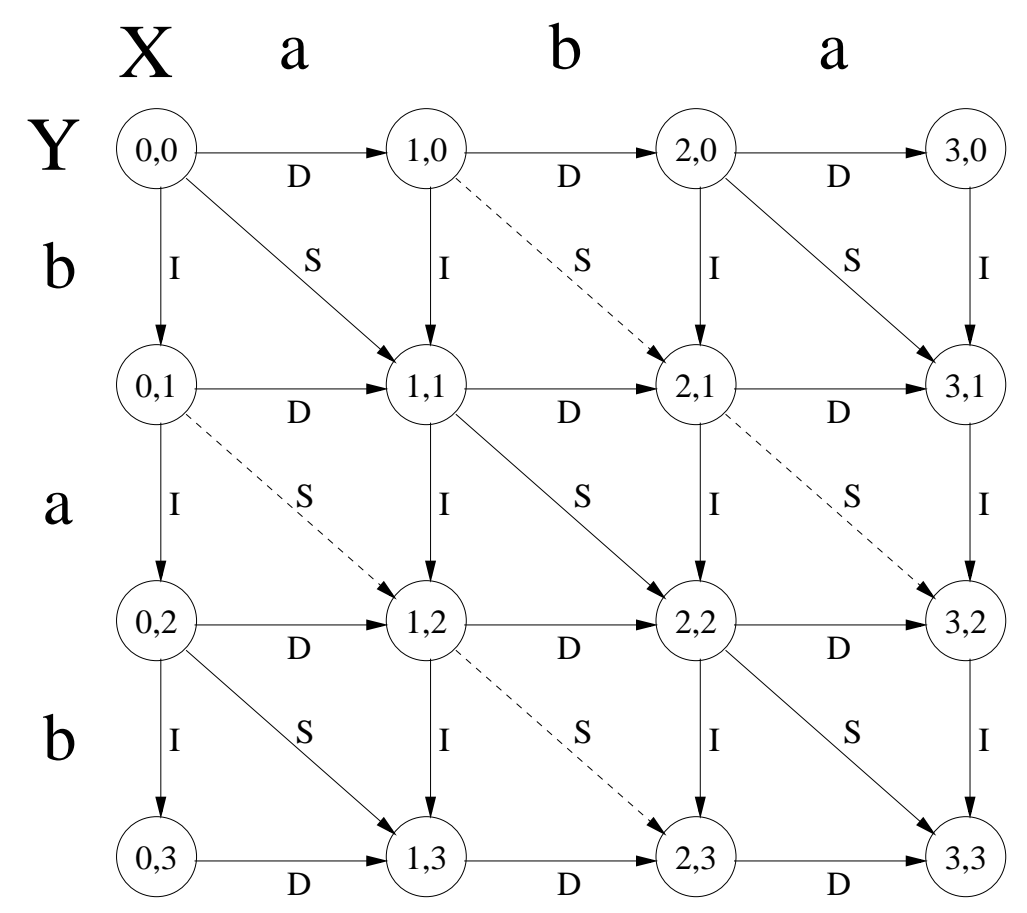

Figure 1. The edit graph $G_{X, Y}$ for the strings $X=a b a$ and $Y=b a b$.

the weights of vertical, horizontal, matching diagonal, and non-matching diagonal arcs are given by $\gamma_{I}, \gamma_{D}, \gamma_{M}, \gamma_{N}$, respectively.

The edit distance $E D_{X, Y, \gamma}$ is the weight of a shortest edit path in $G_{X, Y}$. In other words, let $\mathcal{P}=\mathcal{P}_{X, Y}$ denote the set of all edit paths between $X$ and $Y$ in $G_{X, Y}$. If $W_{\gamma}(p)$ denotes the sum of the weights of the $\operatorname{arcs}$ in $p \in \mathcal{P}$ ( $W_{\gamma}$ is called the path-weight function corresponding to $\gamma$ ), then

$$
E D_{X, Y, \gamma}=\min _{p \in \mathcal{P}} W_{\gamma}(p) .
$$

The normalized edit distance $N E D_{X, Y, \gamma}$ is the minimum amortized weight of paths in $\mathcal{P}$. That is, if $L$ denotes the path-length function which gives the number of arcs in $p \in \mathcal{P}$, then

$$
N E D_{X, Y, \gamma}=\min _{p \in \mathcal{P}} \frac{W_{\gamma}(p)}{L(p)} .
$$

$N E D_{X, Y, \gamma}$ is undefined when $L(p)=0$ which happens only when both $X$ and $Y$ are null. Figure 2 shows optimal ordinary and normalized edit paths $p_{1}$ and $p_{2}$ for the graph $G_{X, Y}$ of Figure 1, for $\gamma=(9,7,0,5)$. Note that $W_{\gamma}\left(p_{1}\right) / L\left(p_{1}\right)=5$ whereas the optimum value of (2) is $W_{\gamma}\left(p_{2}\right) / L\left(p_{2}\right)=4$.

Given strings $X$ and $Y$, let $\mathcal{F}=\left\{\left(h(p), v(p), d_{M}(p)\right.\right.$, $\left.\left.d_{N}(p)\right) \mid p \in \mathcal{P}\right\}$ where $h(p), v(p), d_{M}(p)$, and $d_{N}(p)$ denote respectively the number of horizontal, vertical, matching diagonal, and non-matching diagonal arcs in $p . W_{\gamma}$ and
$L$ can be thought of as linear functions from $\mathcal{F}$ to the real numbers. For a given $p \in \mathcal{P}$

$$
\begin{aligned}
W_{\gamma}(p)= & \gamma_{D} h(p)+\gamma_{I} v(p) \\
& +\gamma_{M} d_{M}(p)+\gamma_{N} d_{N}(p), \\
L(p)= & h(p)+v(p)+d_{M}(p)+d_{N}(p) .
\end{aligned}
$$

From the structure of the graph $G_{X, Y}$ we have

$$
\begin{aligned}
m & =h(p)+d_{M}(p)+d_{N}(p), \\
n & =v(p)+d_{M}(p)+d_{N}(p) .
\end{aligned}
$$

Therefore we can rewrite $W_{\gamma}(p)$ and $L(p)$ as linear functions of two variables $d_{M}(p)$ and $d_{N}(p)$ only, by using the expressions $h(p)=m-d_{M}(p)-d_{N}(p)$, and $v(p)=$ $n-d_{M}(p)-d_{N}(p)$ in (3). Consequently the NED problem becomes the minimization problem stated in (2) in which the numerator and the denominator are given by the linear functions

$$
\begin{aligned}
W_{\gamma}(p)= & m \gamma_{D}+n \gamma_{I} \\
& +\left(\gamma_{M}-\gamma_{I}-\gamma_{D}\right) d_{M}(p) \\
& +\left(\gamma_{N}-\gamma_{I}-\gamma_{D}\right) d_{N}(p) \\
L(p)= & m+n-d_{M}(p)-d_{N}(p)
\end{aligned}
$$




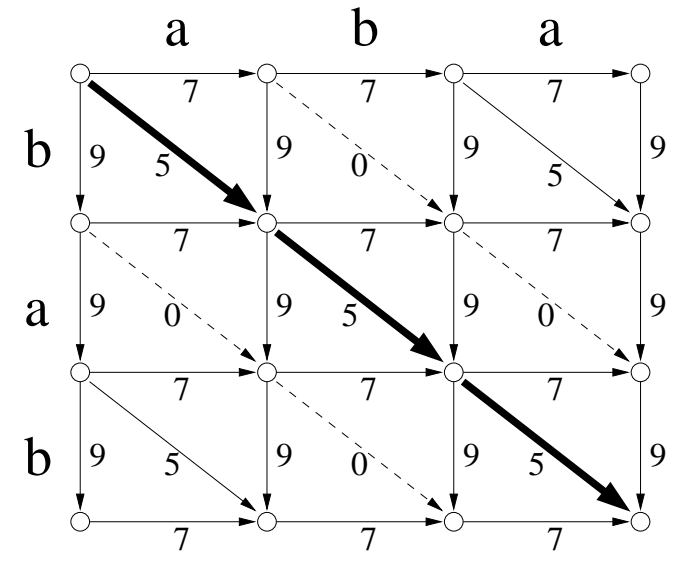

(a)

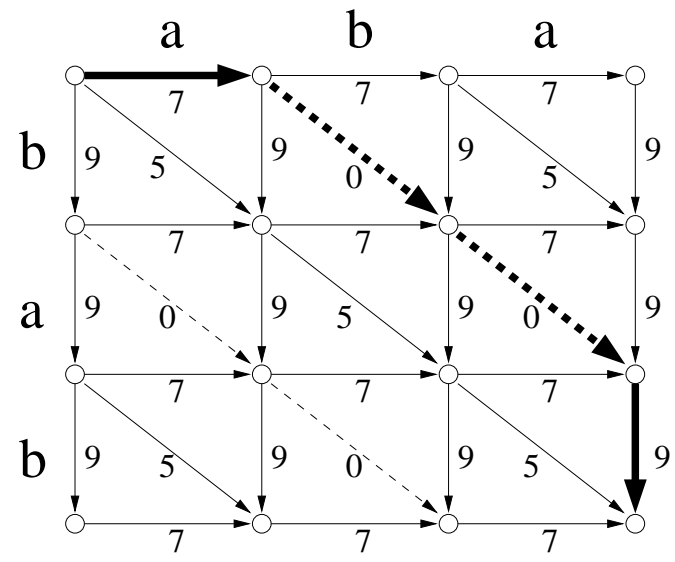

(b)

Figure 2. (a) The optimum edit path $p_{1}$ with weight $15, E D_{X, Y, \gamma}=15$. (b) An optimal edit path $p_{2}$ with amortized weight $16 / 4=4, N E D_{X, Y, \gamma}=4$.

\section{Optimizing the Ratio of Two Linear Func- tions}

For $z=\left(z_{1}, z_{2}, \ldots, z_{n}\right) \in D$ for some domain $D \subseteq \mathbb{R}^{n}$, let $A$ the problem of minimizing $c_{0}+c_{1} z_{1}+\cdots+c_{n} z_{n}$ and let $B$ be the problem of minimizing $\left(a_{0}+a_{1} z_{1}+\cdots+a_{n} z_{n}\right) /\left(b_{0}+b_{1} z_{1}+\cdots+b_{n} z_{n}\right)$ where the denominator is assumed to be always positive in $D$. For $\lambda$ real, let $A(\lambda)$ denote the parametric problem of minimizing $\left(a_{0}+a_{1} z_{1}+\cdots+a_{n} z_{n}\right)-\lambda\left(b_{0}+b_{1} z_{1}+\cdots+b_{n} z_{n}\right)$ in $D$. This is problem $A$ with $c_{i}=a_{i}-\lambda b_{i}, i=0,1, \ldots, n$.

Problem A: minimize $c_{0}+c_{1} z_{1}+\cdots+c_{n} z_{n}$ s.t. $z \in D$

Problem B: minimize $\frac{a_{0}+a_{1} z_{1}+\cdots+a_{n} z_{n}}{b_{0}+b_{1} z_{1}+\cdots+b_{n} z_{n}}$ s.t. $z \in D$

$\operatorname{Problem~A}(\lambda)$ : minimize $\left[\left(a_{0}+a_{1} z_{1}+\cdots+a_{n} z_{n}\right)\right.$ s.t. $z \in D$

$$
\left.-\lambda\left(b_{0}+b_{1} z_{1}+\cdots+b_{n} z_{n}\right)\right]
$$

Dinkelbach's algorithm [3] can be used to solve $B$ when a solution for A is available. Dinkelbach's algorithm uses the parametric method of an optimization technique known as fractional programming. This method is applicable to optimization problems involving ratios of functions which are not necessarily linear. The thesis of the parametric method is that an optimal solution to $B$ can be achieved via the solution of $A(\lambda)$. In fact

$\lambda^{*}$ is the optimum value of $B$ iff the optimum value of $A\left(\lambda^{*}\right)$ is zero .

Dinkelbach's algorithm starts with an initial value for $\lambda$ and repeatedly solves $A(\lambda)$. At each instance of the parametric problem, an optimal solution $z$ of $A(\lambda)$ yields a ratio for $B$. This new ratio is either equal to $\lambda$, in which case it is optimum, or better than $\lambda$ in terms of the objective ratio function of $B$. If it is equal to $\lambda$ then the algorithm terminates. Otherwise, the ratio is taken to be the new value of $\lambda$ and $A(\lambda)$ is solved again. It can be shown that when continued in this fashion, this algorithm takes finitely many steps to find an optimal solution to $B$ if $D$ is a finite set. Furthermore even if $D$ is not finite, convergence to the optimum value $\lambda^{*}$ is guaranteed to be superlinear. Various properties of Dinkelbach's algorithm and fractional programming can be found in $[2,3,6,14]$.

Megiddo [9] introduced a general technique to develop an algorithm for $B$ given an algorithm for $A$. The resulting algorithm for $B$ is the algorithm for $A$ with $c_{i}=a_{i}-\lambda b_{i}$, for $i=0,1, \ldots, n$, where $\lambda$ is treated as a variable, not a constant. That is, the algorithm is the same algorithm as that for $A$ except that the coefficients are not simple constants but linear functions of the parameter $\lambda$. Instead of repeatedly solving $A(\lambda)$ with improved values of $\lambda$, this alternative solution simulates the algorithm for $A$ over these coefficients. The assumption is that the operations among coefficients in the algorithm for $A$ are limited to comparisons and additions. Additions of linear functions are linear and can be computed immediately, but comparisons among linear functions need to be done with some care. The algorithm needs to keep track of the interval in which the optimum value $\lambda^{*}$ of $B$ lies. This is essential because comparisons in the algorithm for $A$ now correspond to those among 
linear functions, and outcomes may vary depending on interval under consideration for $\lambda$.

The algorithm starts with the initial interval $[-\infty,+\infty]$ for $\lambda^{*}$. If the functions to be compared intersect, then their intersection point $\lambda^{\prime}$ determines two subintervals of the initial interval. In calculating which of the two subintervals contains $\lambda^{*}$, algorithm for $A$ is called for help, and the problem $A\left(\lambda^{\prime}\right)$ is solved. The new interval and the result of the comparison are determined from the sign of the optimum value $v$ of $A\left(\lambda^{\prime}\right)$ as will be explained later. With Megiddo's technique, if $A$ is solvable using $O(p(n))$ comparisons and $O(q(n))$ additions then $B$ can be solved in time $O(p(n)(p(n)+q(n))$. We refer the reader to Megiddo's paper [9] for the details of this approach.

Megiddo also showed that for some problems the critical values of $\lambda$ which affect the outcome of comparisons can be precomputed. In such cases the critical values of $\lambda$ give us the possible candidates for the endpoints of the smallest interval which eventually contains the optimum value $\lambda^{*}$. Whenever this can be done, binary search can be used to find $\lambda^{*}$ as follows: Suppose that $v$ is the optimum value of $A(\lambda)$. If $v=0$, then $\lambda$ is the optimum value of $B$, and an optimal solution $z$ of $A(\lambda)$ is also an optimal solution of $B$. On the other hand, if $v>0$, then a larger $\lambda$, and if $v<0$, then a smaller $\lambda$ should be tested (i.e. problem $A(\lambda)$ should be solved with a different value of $\lambda$ ). This procedure continues until the "correct" value $\lambda^{*}$ is found. Let $\lambda^{\prime}$ be the smallest value in the set for which the optimum value of $A\left(\lambda^{\prime}\right)$ is greater than or equal to zero. Then an optimal solution $z$ of $A\left(\lambda^{\prime}\right)$ yields the optimum value $\lambda^{*}$ of $B$. Fewer number of invocations of algorithm $A$ may reduce the time complexity of solving $B$ significantly, which is the case in problems such as minimum ratio cycles, and minimum ratio spanning trees.

In the case of edit distances, problem $A$ is the ordinary edit distance problem, and problem $B$ is the problem of NED in view of our formulation (1), (2), and (4). As we show below the set of possible values of $\lambda$ required for solving $N E D_{X, Y, \gamma}$ can be precomputed efficiently.

Proposition 1 For not both $m$ and $n$ equal to zero, let

$$
Q=\{q(r, s) \mid r, s \text { are non-negative integers, }
$$

$$
\text { and } r+s \leq \min \{m, n\}\}
$$

where $q(r, s)=$

$$
\frac{m \gamma_{D}+n \gamma_{I}+\left(\gamma_{M}-\gamma_{I}-\gamma_{D}\right) r+\left(\gamma_{N}-\gamma_{I}-\gamma_{D}\right) s}{m+n-r-s} .
$$

Then

1. $|Q|=O\left(n^{2}\right)$,

2. For any two strings $X$ and $Y$ over $\Sigma$ of lengths $m$ and $n,\left\{W_{\gamma}(p) / L(p) \mid p \in \mathcal{P}_{X, Y}\right\} \subseteq Q$,

\section{For all $\lambda \in Q, \lambda \geq 0$.}

That is, the possible amortized weights for the paths in $\mathcal{P}_{X, Y}$ are all included in the set $Q$ whose cardinality is $O\left(n^{2}\right)$ (assuming $m \geq n$ ), and whose elements are nonnegative.

Proof Since $r+s \leq \min \{m, n\} \leq n$, by definition $q(r, s)$ takes on $O\left(n^{2}\right)$ distinct values, proving the result about the size of $Q$. The proof of inclusion of all amortized weights in $Q$ directly follows from the definitions of $W_{\gamma}$ and $L$. For non-negative integers $r, s$ with $r+s \leq \min \{m, n\}$, it is easy to see that there are strings $X=x_{1} x_{2} \cdots x_{m}, Y=$ $y_{1} y_{2} \cdots y_{n}$ and an edit path $p$ in $\mathcal{P}_{X, Y}$ such that $d_{M}(p)=r$, $d_{N}(p)=s$, and $q(r, s)$ is the amortized weight of the path $p$ provided that not both $m$ and $n$ are zero. Therefore, the set $Q$ in the proposition is actually the union of the ratios $\left\{W_{\gamma}(p) / L(p) \mid p \in \mathcal{P}_{X, Y}\right\}$ where $X$ and $Y$ vary over all strings of length $m$ and $n$ over $\Sigma$. Since amortized weights are non-negative for the paths in $P_{X, Y}, Q$ does not include a negative number.

\section{The Algorithm}

We propose the following algorithm UniformNED to solve the NED problem: The algorithm first generates the set of numbers $Q$ which includes all possible amortized weights, i.e. potential optimal values of $N E D_{X, Y, \gamma}$ as described in proposition 1. Next, the optimum value $\lambda^{*}$ is sought in this set by simulating a binary search. At each iteration, the median of the current set is found, and with this value a parametric edit distance problem instance is created. This parametric problem is solved using an ordinary edit distance algorithm. If the optimum value of the parametric problem is zero then the median is the optimum value of $N E D_{X, Y, \gamma}$, if it is negative then the search needs to be directed to smaller values; otherwise if it is positive, the search space is reduced to larger values. In either case, the non-optimal half is removed from the set. The search terminates with the smallest value in the set for which the optimum value of the parametric edit distance problem is zero. This final value is returned as the optimum value of $N E D_{X, Y, \gamma}$. An essential feature of algorithm UniformNED is that the parametric problem created at each iteration is an instance of the ordinary edit distance problem for some cost function $\gamma^{\prime}$ of non-negative weights.

The main steps of the algorithm are shown in Figure 3. The algorithm is clearly correct when $m$ and/or $n$ is zero; otherwise the correctness follows from the facts that all possible amortized weights are included in $Q$; for any $\lambda \in Q$, $E D_{X, Y, \gamma}(\lambda)=0$ if and only if $\lambda \in\left\{W_{\gamma}(p) / L(p) \mid p \in\right.$ $\left.\mathcal{P}_{X, Y}\right\}$; and the smallest $\lambda$ such that $E D_{X, Y, \gamma}(\lambda)=0$ is returned as required. Note that, in the latter case termination is guaranteed since $\lambda \in\left\{W_{\gamma}(p) / L(p) \mid p \in \mathcal{P}_{X, Y}\right\} \neq \varnothing$. 


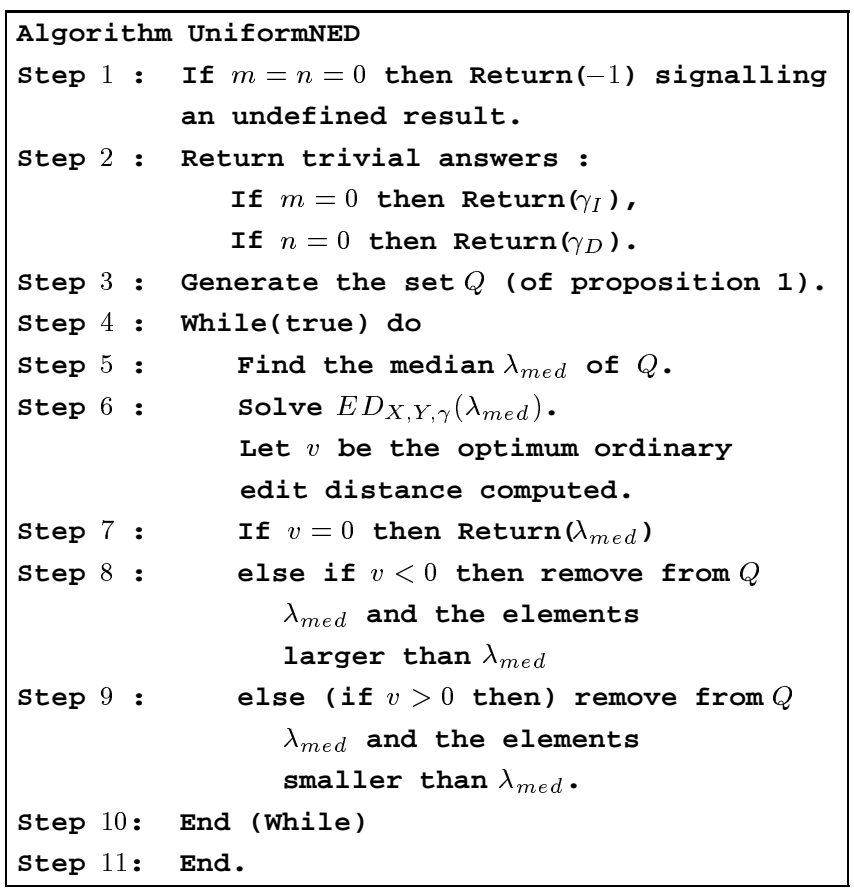

Figure 3. NED algorithm for uniform weights.

Proposition 2 Each parametric edit distance problem $E D_{X, Y, \gamma}(\lambda)$ in algorithm UniformNED can be formulated in terms of the ordinary edit distance problem.

Proof For any $\lambda \in Q, E D_{X, Y, \gamma}(\lambda)$ is the minimization problem $\min _{p \in \mathcal{P}}\left[W_{\gamma}(p)-\lambda L(p)\right]$. We calculate that

$$
\begin{aligned}
& \min _{p \in \mathcal{P}}[\left.W_{\gamma}(p)-\lambda L(p)\right] \\
&=\min _{p \in \mathcal{P}}[ {\left[m \gamma_{D}+n \gamma_{I}\right.} \\
&+\left(\gamma_{M}-\gamma_{I}-\gamma_{D}\right) d_{M}(p) \\
&+\left(\gamma_{N}-\gamma_{I}-\gamma_{D}\right) d_{N}(p) \\
&\left.-\lambda\left(m+n-d_{M}(p)-d_{N}(p)\right)\right] \\
&=\quad \min _{p \in \mathcal{P}} \quad\left(m \gamma_{D}+n \gamma_{I}\right. \\
&+\left(\gamma_{M}+\lambda-\gamma_{I}-\gamma_{D}\right) d_{M}(p) \\
&\left.+\left(\gamma_{N}+\lambda-\gamma_{I}-\gamma_{D}\right) d_{N}(p)\right] \\
&=-\lambda(m+n) \\
& E D_{X, Y, \gamma^{\prime}}-\lambda(m+n)
\end{aligned}
$$

where $\gamma^{\prime}=\left(\gamma_{I}, \gamma_{D}, \gamma_{M}+\lambda, \gamma_{N}+\lambda\right)$. Since $\lambda$ is nonnegative for all $\lambda \in Q, \gamma^{\prime}$ includes no negative weights. Hence we have a valid instance of the ordinary edit distance problem with cost function $\gamma^{\prime}$.

Corollary 1 Any ordinary edit distance algorithm A can be used to compute a parametric problem $E D_{X, Y, \gamma^{\prime}}$ in algorithm UniformNED, provided that $\gamma^{\prime}$ does not conflict with $A$ 's assumption on cost functions.
Theorem 1 If parametric edit distance problems $E D_{X, Y, \gamma^{\prime}}$ are solvable by an algorithm $A$ where $\gamma^{\prime}=\left(\gamma_{I}, \gamma_{D}, \gamma_{M}+\right.$ $\left.\lambda, \gamma_{N}+\lambda\right)$ for $\lambda \in Q$ and if $C(m, n)$ denotes the time complexity of algorithm $A$, then $N E D_{X, Y, \gamma}$ can be computed in time $O\left(n^{2}\right)+C(m, n) O(\log n)$.

Proof We show that this complexity result can be achieved by using algorithm UniformNED. Step 3 of the algorithm takes $O\left(n^{2}\right)$ time. The while loop iterates $O(\log n)$ times. If linear-time median finding algorithm [1] is used in step 5 , then the total time (from start to completion of the loop) spent in steps 8 , and 9 is $O\left(n^{2}\right)$. Solving a parametric problem in step 6 , which is an ordinary edit distance computation problem by proposition 2 , takes $C(m, n)$ time. The remaining steps take constant time. Thus the resulting time complexity is as expressed in the theorem.

Wagner and Fisher's $O(m n)$-time edit distance algorithm [17] can be used to compute the shortest edit paths in $G_{X, Y}$. This algorithm is suitable as algorithm $A$ in UniformNED to solve the parametric ordinary edit distance problems, since the $O(m n)$ time complexity is independent of the cost function. Since $C(m, n)=O(m n)$ in this case, we have

Corollary 2 Algorithm UniformNED computes the normalized edit distance $N E D_{X, Y, \gamma}$ using $O(m n \log n)$ operations.

\section{Implementation Details}

Termination of UniformNED requires absolute accuracy in ordinary edit distance computations. Errors associated with floating point operations may be problematic. This can be overcome by modifying the algorithm as shown in Figure 4.

Assuming that there is no sign-error in the result of ordinary edit distance computation, the optimum value will be returned either in step 7 or after the termination of the while loop, since it will not be removed from $Q$. Therefore termination is guaranteed. The time complexity of UniformNED2 is the same as that of UniformNED.

If an optimal edit path is also desired, an additional parametric edit distance problem needs to be solved. An optimal edit path for the parametric problem $E D_{X, Y, \gamma}\left(N E D_{X, Y, \gamma}\right)$ has amortized weight $N E D_{X, Y, \gamma}$. Therefore, following the computation of $N E D_{X, Y, \gamma}$ we need to solve $E D_{X, Y, \gamma}\left(N E D_{X, Y, \gamma}\right)$ for an optimal edit path. Note that an optimal edit path may not be computed correctly by modifying UniformNED2 such that the edit path returned by $E D_{X, Y, \gamma}\left(\lambda_{m e d}\right)$ (along with the optimum edit distance value) is saved in step 6 , and returned in step 7 and 11, since it is possible that with the last (remaining) element in $Q$ no parametric ordinary edit distance problem has been 


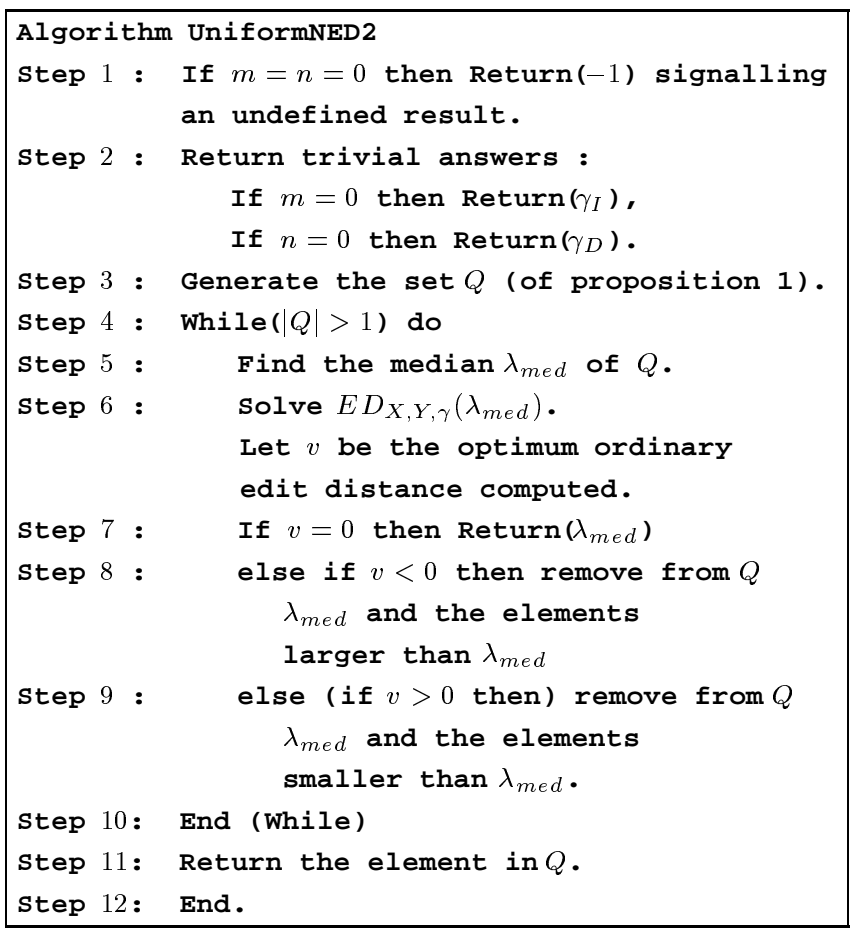

Figure 4. Modified NED algorithm for uniform weights.

solved, in which case the element returned is not the amortized weight of the edit path stored.

\section{Remarks}

There are alternate ways of formulating $N E D_{X, Y, \gamma^{\prime}}$ in terms of shortest edit paths if we relax certain conditions. For example, it is possible to write

$$
N E D_{X, Y, \gamma^{\prime}}=\min _{p \in \mathcal{P}} W_{\gamma^{\prime}}(p)
$$

where $\gamma^{\prime}=\left(\gamma_{I}-\lambda, \gamma_{D}-\lambda, \gamma_{M}-\lambda, \gamma_{N}-\lambda\right)$ as used in [16]. However in such a formulation, $\gamma^{\prime}$ may include a negative weight, resulting in a non-standard instance of the ordinary edit distance problem that needs to be solved as part of UniformNED.

We have formulated algorithm UniformNED in such a way that any ordinary edit distance algorithm $A$ can be used to compute a parametric problem $E D_{X, Y, \gamma^{\prime}}$ of Step 6, as long as $A$ is capable of solving $E D_{X, Y, \gamma^{\prime}}$ as indicated in corollary 1 . This property of $A$ needs to be kept in mind in the time complexity $O\left(n^{2}\right)+C(m, n) O(\log n)$ of theorem 1. In other words, not every fast algorithm $A$ for the ordinary edit distance calculation may be a suitable candidate for $A$, if it is not general enough to meet the conditions in corollary 1. For example edit distance algorithms which assume a fixed cost function (e.g. $\gamma=(1,1,0,1)$ ) and achieve fast running times by using this constant nature of the weights $[15,10]$ are evidently not suitable for UniformNED. This is because each execution of Step 6 of the algorithm uses a different cost function $\gamma^{\prime}$.

Masek and Paterson [8] gave a $C(m, n)=$ $O(m n / \log n)$-time algorithm which can be used as algorithm $A$ here, but it is no longer true that the running time of UniformNED is as indicated by theorem 1. The assumption in the algorithm of Masek and Paterson is that the weights are integral multiples of a positive real constant $r$. We can easily show that if the assumption is true for $\lambda$, then it is also true for $\lambda^{\prime}$ that arises in UniformNED. Suppose that $\gamma_{I}, \gamma_{D}, \gamma_{M}$, and $\gamma_{N}$ are all integer multiples of a real positive number $r$ and let $\lambda$ be in $Q$. If $\lambda=0$, then the assertion is true for the weights in $\gamma^{\prime}$, since $\gamma^{\prime}=\gamma$ in this case. If $\lambda>0$ then there exist positive integers $a$, and $b$ such that $\lambda=a r / b$ because of the way we generate the set $Q$ in proposition 1. Therefore in this case, $\gamma_{I}, \gamma_{D}$, $\gamma_{M}+\lambda$, and $\gamma_{N}+\lambda$ are integer multiples of the positive real number $r / b$. There appear to be no other obvious choice of the new constant such that the assumption holds for $\gamma^{\prime}$. But the algorithm of Masek and Paterson takes time proportional to some function of $1 / r$ which normally is absorbed in the "big O", since it is independent of $m$ and $n$. The discrepancy factor among the magnitude of constants for different parametric problems of UniformNED can be in order of $m$ since $b$ has to be, in some cases, as large as the maximum path length $m+n$. Therefore even though it may be possible to achieve $O(m n / \log n)$ with $\gamma$, solving some parametric problems using this $A$ would take significantly (by about a factor of $m$ ) more time.

Ukkonen's $O(d n)$-time output-size sensitive algorithm [15], where $d$ is the actual edit distance, does not seem applicable because it assumes that the weights are nonnegative and they fulfill the triangle inequality, that is, in the case of uniform weights, for a given cost function $\gamma=\left(\gamma_{D}, \gamma_{I}, \gamma_{M}, \gamma_{N}\right), a \leq b+c$ for all $a, b, c$ $\in\left\{\gamma_{D}, \gamma_{I}, \gamma_{M}, \gamma_{N}\right\}$. Even though the parametric problems of UniformNED can be formulated in terms of conventional edit distance problem with different cost functions $\gamma^{\prime}$, the triangle inequality cannot always be met while simultaneously keeping the weights non-negative. For example, setting $\gamma^{\prime}=\left(\gamma_{I}-\lambda, \gamma_{D}-\lambda, \gamma_{M}-\lambda, \gamma_{N}-\lambda\right)$ maintains the triangle inequality by allowing negative weights, or setting $\gamma^{\prime}=\left(\gamma_{I}, \gamma_{D}, \gamma_{M}+\lambda, \gamma_{N}+\lambda\right)$ guarantees non-negativity of weights, by possibly destroying the triangle inequality.

\section{Conclusion}

In the absence of theoretical time complexity results for the application of fractional programming to normalized edit distance calculations, we have improved the time 
complexity of normalized edit distance computation from $O\left(m n^{2}\right)$ to $O(m n \log n)$ when the weights of edit operations are uniform. Although this result is of theoretical interest, we believe that the real applications will be in favor of fractional programming normalized edit distance algorithm because of its easy implementation, observed experimental performance, and the generality of the cost function that can be used.

The worst-case and the expected time complexity of fractional programming formulation of NED need to be investigated for a better assessment of the value of the algorithm presented in this paper, keeping in mind that our $O(m n \log n)$ bound is in the worst-case. It has already been noted that fractional programming based distance calculations give good experimental results. It seems that while our algorithm has an improved worst-case time complexity, fractional programming normalized edit distance algorithm may actually be provably faster on the average.

\section{References}

[1] M. Blum, R. W. Floyd, R. Pratt, R. L. Rivest, and R. E. Tarjan. Time bounds for selection. Journal of Computer and System Sciences, 7(4):448-461, August 1973.

[2] B. D. Craven. Fractional Programming. Helderman Verlag, Berlin, 1988.

[3] W. Dinkelbach. On nonlinear fractional programming. Management Science, 13(7):492-498, March 1967.

[4] Ö. Egecioḡlu and M. Ibel. Parallel algorithms for fast computation of normalized edit distances. Proceedings. Eighth IEEE Symposium on Parallel and Distributed Processing (SPDP'96), pages 496-503, October 1996.

[5] Z. Galil and R. Giancarlo. Data structures and algorithms for approximate string matching. Journal of Complexity, 4(1):33-72, March 1988.

[6] T. Ibaraki. Parametric approaches to fractional programs. Mathematical Programming, 26(3):345-362, August 1983.

[7] A. Marzal and E. Vidal. Computation of normalized edit distances and applications. IEEE Transactions on Pattern Analysis and Machine Intelligence, 15(9):926-932, September 1993.

[8] W. J. Masek and M. S. Paterson. A faster algorithm computing string edit distances. Journal of Computer and System Sciences, 20(1):18-31, February 1980.

[9] N. Megiddo. Combinatorial optimization with rational objective functions. Mathematics of Operations Research, 4(4):414-424, November 1979.

[10] E. W. Myers. An O(ND) difference algorithm and its variations. Algorithmica, 1(2):251-266, 1986.

[11] B. J. Oommen and K. Zhang. The normalized string editing problem revisited. IEEE Transactions on Pattern Analysis and Machine Intelligence, 18(6):669-672, June 1996.

[12] S. V. Rice, H. Bunke, and T. A. Nartker. Classes of cost functions for string edit distance. Algorithmica, 18(2):271280, 1997.
[13] D. Sankoff and J. B. Kruskal. Time Warps, String Edits, and Macromolecules: The Theory and Practice of Sequence Comparison. Addison-Wesley, Reading, MA, 1983.

[14] M. Sniedovich. Dynamic Programming. Marcel Dekker, New York, 1992.

[15] E. Ukkonen. Algorithms for approximate string matching. Information and Control, 64:100-118, 1985.

[16] E. Vidal, A. Marzal, and P. Aibar. Fast computation of normalized edit distances. IEEE Transactions on Pattern Analysis and Machine Intelligence, 17(9):899-902, September 1995.

[17] R. A. Wagner and M. J. Fisher. The string-to-string correction problem. Journal of the Association for Computing Machinery, 21(1):168-173, January 1974. 